\title{
Study on the Relationship between Manganese Concentrations in Rural Drinking Water and Incidence and Mortality Caused by Cancer in Huai'an City
}

\author{
Qin Zhang, Enchun Pan, ${ }^{1}$ Linfei Liu, ${ }^{1}$ Wei Hu, ${ }^{1}$ Yuan He, ${ }^{1}$ Qiujin Xu, ${ }^{3}$ and Cunzhen Liang ${ }^{4}$ \\ ${ }^{1}$ Huaian City Center for Disease Prevention and Control, Huaian, Jiangsu 223001, China \\ ${ }^{2}$ The Department of No-Communicable Diseases Control and Prevention, Huai'an City Center for Disease Prevention and Control, \\ 118 Huaihai North Road, Huaian, Jiangsu 22300, China \\ ${ }^{3}$ China Research Academy of Environmental Science, Beijing 100012, China \\ ${ }^{4}$ Department of Environmental Engineering, Beijing Petrochemical College, Beijing 102617, China
}

Correspondence should be addressed to Enchun Pan; hypec@163.com

Received 14 February 2014; Accepted 30 July 2014; Published 3 November 2014

Academic Editor: Xin-yuan Guan

Copyright (c) 2014 Qin Zhang et al. This is an open access article distributed under the Creative Commons Attribution License, which permits unrestricted use, distribution, and reproduction in any medium, provided the original work is properly cited.

Background. Cancer is a significant disease burden in the world. Many studies showed that heavy metals or their compounds had connection with cancer. But the data conflicting about the relationship of manganese $(\mathrm{Mn})$ to cancer are not enough. In this paper, the relationship was discussed between $\mathrm{Mn}$ concentrations in drinking water for rural residents and incidence and mortality caused by malignant tumors in Huaian city. Methods. A total of 158 water samples from 28 villages of 14 towns were, respectively, collected during periods of high flow and low flow in 3 counties of Huaian city, along Chinese Huai'he River. The samples of deep groundwater, shallow groundwater, and surface water were simultaneously collected in all selected villages. Mn concentrations in all water samples were determined by inductively coupled plasma-mass spectrometry (ICP-MS 7500a). The correlation analysis was used to study the relationship between the Mn concentration and cancer incidence and mortality. Results. Mn concentrations detectable rate was $100 \%$ in all water samples. The mean concentration was $452.32 \mu \mathrm{g} / \mathrm{L} \pm 507.76 \mu \mathrm{g} / \mathrm{L}$. There was significant difference between the high flow period and low flow period $(t=-5.23, P<0.05)$ and also among deep groundwater, shallow groundwater, and surface water $(F=5.02, P<0.05)$. The ratio of superscale of $\mathrm{Mn}$ was $75.32 \%$. There was significant difference of $\mathrm{Mn}$ level between samples in the high flow period and low flow period $\left(\chi^{2}=45.62, P<0.05\right)$ and also among deep groundwater, shallow groundwater, and surface water $\left(\chi^{2}=10.66, P<0.05\right)$. And also we found that, during the low flow period, Mn concentration has positive correlation with cancer incidence and mortality; for a $1 \mu \mathrm{g} / \mathrm{L}$ increase in Mn concentration, there was a corresponding increase of $0.45 / 100000$ new cancer cases and $0.35 / 100000$ cancer deaths $(P<0.05)$. Conclusions. In Huai'an city, the mean concentration of $\mathrm{Mn}$ in drinking water was very high. Mn concentration correlated with cancer incidence and mortality.

\section{Background}

The global burden of cancer is increasing largely as a result of population aging and growth. Based on the GLOBOCAN 2008 estimates, about 12.7 million cancer cases and 7.6 million cancer deaths are estimated to have occurred in 2008 [1]. Cancer is also one of the leading causes of deaths in China; it remains a significant disease burden. The crude incidence in Chinese cancer registration areas was $285.91 / 10^{5}$, and the crude mortality was $180.54 / 10^{5}$ [2]. The complex pathogenesis of cancer is considered as the interaction between environment exposures and genetics with multiple factors and steps. It is reported that environmental factors are the most important factors for cancer, especially, chemical factors which up to $80 \%-90 \%$ [3]. Huaian city lies downstream of Huaihe Downriver River and has a higher cancer incidence which has been taken into account $[4,5]$.

Manganese ( $\mathrm{Mn})$ is widely distributed as one of the most abundant elements in the earth's crust and also in many foodstuffs. $\mathrm{Mn}$ is an essential trace element with many biological functions but toxic at higher doses $[6,7]$. In industry, the majority of $\mathrm{Mn}$ is used to make alloys and steel. 
Interest in environmental manganese has increased recently while $\mathrm{Mn}$ is replacing lead as an antiknock agent in gasoline globally in the form of methylcyclopentadienyl manganese tricarbonyl (MMT) [8,9]. A number of metals and metal compounds have been classified by the International Agency for Research on Cancer (IARC) as known carcinogens, but $\mathrm{Mn}$ and its compounds have not been evaluated [10]. Many studies show that $\mathrm{Mn}$ has an important role in neurodegenerative diseases [11]. But the data conflicting about the relationship of manganese to cancer are not enough. In this paper, we mainly discussed the relationship between Mn concentrations in drinking water for rural residents and incidence and mortality caused by malignant tumors.

\section{Materials and Methods}

2.1. General Situation of Study Area. Huaian city is located in the center of Jiangsu province across Huaihe River. It has 5.4 millionpeople and $70 \%$ of them are rural residents. It has four counties, four districts, and one economic development zone which contain in all 116 towns. Here it has a high cancer incidence especially digest system neoplasm. Currently, only Huaian city and its urban district use central water supply, while the other towns use small central water supply in rural areas. Before quality improvement, drinking water for rural residents mainly came from well and hand pressure well or earlier from the ditch and pond.

2.2. Water Sample. We chose 5 towns from Jinhu County and Chuzhou county, respectively, and 4 towns from Xuyi County and chose 2 villages with a higher cancer incidence of every chosen town. Finally, there were 28 villages. 158 water samples from these villages were, respectively, collected in two water period: the high flow and low flow period, and three water sources: deep groundwater, shallow groundwater, and surface water. About $500 \mathrm{~mL}$ of water was bottled and $\mathrm{HNO}_{3}$ was added to keep $\mathrm{pH}<2$. Then the samples were transported to the lab and were stored under $4^{\circ} \mathrm{C}$. The water samples were analyzed by ICP-MS 7500a.

2.3. Data Analysis. According to national standards for drinking water quality, Mn concentrations more than $100 \mu \mathrm{g} /$ $L$ were defined as overproof in drinking water. We use SAS9.1 for data analysis. The data of incidence and mortality of population Cancer from 2008 to 2010 year come from Cancer Registration System of Huaian city. The correlation analysis was used to study the relationship between the Mn concentration and cancer incidence and mortality.

\section{Results}

3.1. Incidence and Mortality of Cancer. Cancer incidence and mortality of Chuzhou county, Jinhu county, and Xuyi county was $216.54 / 10^{5}, 282.47 / 10^{5}$, and $179.33 / 10^{5}$ and $157.01 / 10^{5}$, $192.20 / 10^{5}$, and $170.02 / 10^{5}$. There was a significant difference in cancer incidence and mortality among these three counties $(P<0.01)$. This study included 158 water samples from 28 villages of 14 towns in Chuzhou county, Jinhu county, and
TABLE 1: The comparison of Mn concentrations in drinking water in Huai'an city.

\begin{tabular}{lccccc}
\hline Variable & $N$ & Mean & Std. & $t(F)$ & $P$ \\
\hline Counties & & & & & \\
$\quad$ Chuzhou county & 57 & 401.15 & 309.76 & & \\
$\quad$ Jinhu county & 59 & 574.46 & 701.6 & 2.92 & 0.06 \\
$\quad$ Xuyi county & 42 & 350.17 & 352.26 & & \\
Periods & & & & & \\
$\quad$ High flow period & 84 & 265.47 & 388.83 & -5.34 & 0.00 \\
$\quad$ Low flow period & 74 & 664.41 & 544.82 & & \\
Sources & & & & & \\
$\quad$ Surface water & 47 & 506.33 & 362.88 & & \\
$\quad$ Shallow groundwater & 55 & 573.64 & 672.47 & 5.02 & 0.01 \\
$\quad$ Deep groundwater & 56 & 287.83 & 370.25 & & \\
$\quad$ All & 158 & 452.32 & 507.76 & & \\
\hline
\end{tabular}

Xuyi county. All of them, 84 water samples were from the high flow period and 74 water samples from the low flow period. Detectable rate was $100 \%$.

3.2. The Comparison of $\mathrm{Mn}$ Concentrations in Drinking Water. The min concentration of $\mathrm{Mn}$ in water samples was $0.02 \mu \mathrm{g} / \mathrm{L}$, the max was $4364.00 \mu \mathrm{g} / \mathrm{L}$, and the mean was $452.32 \mu \mathrm{g} / \mathrm{L} \pm$ $507.76 \mu \mathrm{g} / \mathrm{L}$. The mean concentration of $\mathrm{Mn}$ in Chuzhou county, Jinhu county, and Xuyi county was, respectively, $401.15 \mu \mathrm{g} / \mathrm{L} \pm 309.76 \mu \mathrm{g} / \mathrm{L}, 574.46 \mu \mathrm{g} / \mathrm{L} \pm 701.60 \mu \mathrm{g} / \mathrm{L}$, and $350.17 \mu \mathrm{g} / \mathrm{L} \pm 352.26 \mu \mathrm{g} / \mathrm{L}$. There was no significant difference in the mean concentration among these three counties $(F=$ 2.92, $P>0.05)$. The mean concentration of $\mathrm{Mn}$ in the high flow period and the low flow period was $265.47 \mu \mathrm{g} / \mathrm{L} \pm$ $388.83 \mu \mathrm{g} / \mathrm{L}$, and $664.41 \mu \mathrm{g} / \mathrm{L} \pm 544.82 \mu \mathrm{g} / \mathrm{L}$, and there was a significant difference between them $(t=-5.34, P<$ 0.05). The mean concentration of $\mathrm{Mn}$ in deep groundwater, shallow groundwater, and surface water was $287.83 \mu \mathrm{g} / \mathrm{L} \pm$ $370.25 \mu \mathrm{g} / \mathrm{L}, 573.64 \mu \mathrm{g} / \mathrm{L} \pm 672.47 \mu \mathrm{g} / \mathrm{L}$, and $506.33 \mu \mathrm{g} / \mathrm{L} \pm$ $362.47 \mu \mathrm{g} / \mathrm{L}$, and there was a significant difference among them $(F=5.02, P<0.05)$. The concentration of $\mathrm{Mn}$ in deep groundwater and shallow groundwater was more than in surface water; the difference was significant $(P<0.05)$. During the high flow period, the mean concentration of $\mathrm{Mn}$ in three water sources was difference significantly with the mean concentration in deep groundwater being only $72.01 \mu \mathrm{g} / \mathrm{L} \pm 130.91 \mu \mathrm{g} / \mathrm{L}$ while the other two sources being $437.85 \mu \mathrm{g} / \mathrm{L} \pm 439.79 \mu \mathrm{g} / \mathrm{L}, 286.56 \mu \mathrm{g} / \mathrm{L} \pm 429.09 \mu \mathrm{g} / \mathrm{L}(F=$ 7.19, $P<0.05$ ) (Table 1).

\subsection{The Comparison of Mn Concentrations in Drinking Water} during Different Period. During high flow period, the mean concentration of $\mathrm{Mn}$ in deep groundwater, shallow groundwater, and surface water was $437.85 \mu \mathrm{g} / \mathrm{L} \pm 439.79 \mu \mathrm{g} / \mathrm{L}$, $286.56 \mu \mathrm{g} / \mathrm{L} \pm 429.09 \mu \mathrm{g} / \mathrm{L}$, and $72.01 \mu \mathrm{g} / \mathrm{L} \pm 130.91 \mu \mathrm{g} / \mathrm{L}$, and there was a significant difference among them $(F=7.19$, $P<0.05)$. During high flow period, the mean concentration of $\mathrm{Mn}$ in deep groundwater, shallow groundwater, and surface water was $607.24 \mu \mathrm{g} / \mathrm{L} \pm 168.38 \mu \mathrm{g} / \mathrm{L}, 871.35 \mu \mathrm{g} / \mathrm{L} \pm$ $753.13 \mu \mathrm{g} / \mathrm{L}$, and $503.65 \mu \mathrm{g} / \mathrm{L} \pm 406.83 \mu \mathrm{g} / \mathrm{L}$, and there was a 
TABle 2: The comparison of Mn concentrations in drinking water during different periods.

\begin{tabular}{|c|c|c|c|c|c|c|}
\hline Periods & Water sources & $N$ & Mean & Std. & $F$ & $P$ \\
\hline \multirow{4}{*}{ High flow period } & Surface water & 28 & 437.85 & 439.79 & \multirow{4}{*}{7.19} & \multirow{4}{*}{0.00} \\
\hline & Shallow groundwater & 28 & 286.56 & 429.09 & & \\
\hline & Deep groundwater & 28 & 72.01 & 130.91 & & \\
\hline & All & 84 & 265.47 & 388.83 & & \\
\hline \multirow{4}{*}{ Low flow period } & Surface water & 19 & 607.24 & 168.38 & \multirow{4}{*}{3.50} & \multirow{4}{*}{0.04} \\
\hline & Shallow groundwater & 27 & 871.35 & 753.13 & & \\
\hline & Deep groundwater & 28 & 503.65 & 406.83 & & \\
\hline & All & 74 & 664.41 & 544.82 & & \\
\hline
\end{tabular}

TABLE 3: The comparison of superscale ratio of $\mathrm{Mn}$ in drinking water.

\begin{tabular}{lcccccc}
\hline \multirow{2}{*}{ Variable } & \multicolumn{2}{c}{ Superscale } & \multicolumn{2}{c}{ Qualified } & \multirow{2}{*}{$\chi^{2}$} & \multirow{2}{*}{$P$} \\
& $N$ & $\%$ & $N$ & $\%$ & & \\
\hline Counties & & & & & & \\
$\quad$ Chuzhou county & 46 & 80.70 & 11 & 19.30 & & \\
$\quad$ Jinhu county & 46 & 77.97 & 13 & 22.03 & 3.86 & 0.15 \\
$\quad$ Xuyi county & 27 & 64.29 & 15 & 35.71 & & \\
Periods & & & & & & \\
$\quad$ High flow period & 45 & 53.57 & 39 & 46.43 & 45.62 & 0.00 \\
$\quad$ Low flow period & 74 & 100.00 & 0 & 0.00 & & \\
$\quad$ Sources & & & & & & \\
$\quad$ Surface water & 41 & 87.23 & 6 & 12.77 & & \\
$\quad$ Shallow groundwater & 44 & 80.00 & 11 & 20.00 & & \\
$\quad$ Deep groundwater & 34 & 60.71 & 22 & 39.29 & & \\
$\quad$ All & 119 & 75.32 & 39 & 24.68 & & \\
\hline
\end{tabular}

significant difference among them $(F=3.50, P<0.05)$. S-N$\mathrm{K}$ showed that in high flow period Mn concentration in both shallow groundwater and surface water were higher than that in deep groundwater $(P<0.05)$. During high flow period, Mn concentration in shallow groundwater was higher than that in deep groundwater $(P<0.05)$ (Table 2$)$.

3.4. The Comparison of Superscale Ratio of $M n$ in Drinking Water. Referring to Standards for Drinking Water Quality, the ratio of superscale of $\mathrm{Mn}$ in 158 water samples was $75.32 \%$. In Chuzhou county, Jinhu county and Xuyi county, the ratio of superscale of Mn was $80.70 \%, 77.97 \%$, and $64.29 \%$, the difference was not significant $\left(\chi^{2}=3.86, P>0.05\right)$. The ratio of superscale of $\mathrm{Mn}$ in the high flow period and the low flow period was $53.57 \%$ and $100 \%$; the difference was significant $\left(\chi^{2}=45.62, P<0.05\right)$. The ratio of superscale in surface water was $87.23 \%$ which was the highest one. And next one was in shallow groundwater which was $80.00 \%$. The third one was in deep groundwater which was $60.71 \%$. The difference among them was significant $\left(\chi^{2}=10.66, P<0.05\right)$ (Table 3$)$.

3.5. The Comparison of Superscale Ratio of Mn in Drinking Water in Different Period. Be worth mentioning, the ratio of superscale in all water sources during the low flow period was $100 \%$. But in high flow period, the ratio of superscale of three water sources was different, and the ratio was $78.57 \%, 60.71 \%$,
TABLE 4: The Comparison of superscale ratio of $\mathrm{Mn}$ in drinking water in different periods.

\begin{tabular}{lcccccc}
\hline \multirow{2}{*}{ Variable } & \multicolumn{2}{c}{ Superscale } & \multicolumn{2}{c}{ Qualified } & \multirow{2}{*}{$\chi^{2}$} & $P$ \\
& $N$ & $\%$ & $N$ & $\%$ & & \\
\hline Sources & & & & & & \\
$\quad$ Surface water & 22 & 78.57 & 6 & 21.43 & & \\
$\quad$ Shallow groundwater & 17 & 60.71 & 11 & 39.29 & 19.24 & 0.00 \\
$\quad$ Deep groundwater & 6 & 21.43 & 22 & 78.57 & & \\
All & 45 & 53.57 & 39 & 46.43 & & \\
\hline
\end{tabular}

TABLE 5: The correlation analysis between the Mn concentration and cancer incidence rate and mortality rate.

\begin{tabular}{lcccc}
\hline \multirow{2}{*}{ Variable } & \multicolumn{2}{c}{ Incidence } & \multicolumn{2}{c}{ Mortality } \\
& $r$ & $P$ & $r$ & $P$ \\
\hline Periods & & & & \\
$\quad$ High flow period & -0.12 & 0.68 & -0.22 & 0.45 \\
$\quad$ Low flow period & 0.71 & 0.01 & 0.67 & 0.01 \\
Sources & & & & \\
$\quad$ Surface water & -0.14 & 0.62 & -0.21 & 0.47 \\
$\quad$ Shallow groundwater & 0.47 & 0.09 & 0.34 & 0.24 \\
$\quad$ Deep groundwater & 0.21 & 0.47 & 0.40 & 0.16 \\
All & 0.20 & 0.21 & 0.17 & 0.27 \\
\hline
\end{tabular}

and $21.43 \%$, respectively. The difference among three water sources was significant $\left(\chi^{2}=19.24, P<0.05\right)$ (Table 4$)$.

3.6. The Correlation Analysis. The correlation analysis indicated that the $\mathrm{Mn}$ concentration has positive correlation with cancer incidence and mortality $(P<0.05)$. Regression analysis showed that, for $1 \mu \mathrm{g} / \mathrm{L}$ increase in Mn concentration, there was a corresponding increase of $0.45 / 10^{5}$ cancer cases and $0.35 / 10^{5}$ cancer deaths $(P<0.05)$ (Tables 5 and 6 ).

\section{Discussion}

Our study showed that the mean concentration of $\mathrm{Mn}$ in drinking water in Huai'an city was much higher than the concentration; refer to standards for drinking water quality. The ratio of superscale is high of $75.32 \%$ and during the low flow period was $100 \%$. We also found that the difference between it in the high flow period and low flow period was significant. For three water sources, the ratio of superscale 
TABLE 6: Regression analysis of correlation between Mn concentration and cancer incidence rate and mortality rate during the low flow period.

\begin{tabular}{lcccccc}
\hline \multirow{2}{*}{ Variable } & \multicolumn{3}{c}{ Incidence } & \multicolumn{3}{c}{ Mortality } \\
& $\beta$ & S.E & $P$ & $\beta$ & S.E & $P$ \\
\hline Mn & 0.45 & 0.13 & 0.005 & 0.35 & 0.11 & 0.008 \\
Intercept & 19.92 & 79.66 & 0.81 & 3.29 & 68.32 & 0.96 \\
\hline
\end{tabular}

of $\mathrm{Mn}$ in surface groundwater and shallow groundwater was higher than deep in water, while the same as the mean concentration. Excessive and prolonged exposure to $\mathrm{Mn}$ resulted from welding and mining, inhalation of combustion products from the antiknock agent in fuel (MMT) and highly concentrated Mn concentrations in ground/well water, and so on [7]. In our investigation, these three counties have great quantity industries, such as machinery manufacturing, alternative construction material, chemical industry, and oil industry. So we deduced that $\mathrm{Mn}$ may come from local industrial pollution.

Another important finding was that Mn concentration in drinking water in Huaian city correlated with cancer incidence and mortality. In our city, for a $1 \mu \mathrm{g} / \mathrm{L}$ increase in $\mathrm{Mn}$ concentration, there was a corresponding increase of $0.45 / 10^{5}$ cancer cases and $0.35 / 10^{5}$ cancer deaths. At first glance, this finding appears to be unbelievable. A large ecological study was carried out in North Carolina, and they also found manganese concentration in groundwater correlate with cancer mortality [12]. There are also some other studies showed the relationship between $\mathrm{Mn}$ and cancer. $\mathrm{Mn}$ is a component of the important antioxidant enzyme, manganese peroxide dismutase (MnSOD) which mitigates the oxidative damage from reactive oxygen species, one pathway thought to participate in carcinogenesis, such as lung cancer and breast cancer $[13,14]$. An inverse relationship between breast cancer and hair manganese was documented $[15,16]$.

Though World Health Organization (WHO) found among the general population, the average estimated Mn intake from drinking water is substantially lower than the intake of Mn from the diet, typically $20 \mu \mathrm{g} / \mathrm{d}$ [17], our findings point out thatmental pollution should be taken into account. Factors such as age, chemical species, dose, route of exposure, and dietary components influence both the absorption and bioavailability of manganese in human [18-21]. Thus the measures for mental pollution prevention and control should be of diversity.

Because data were analyzed at the population level and these same county-level factors may be related to individual adverse health behaviors such as cigarette smoking and obesity that contribute to cancer incidence and mortality, these ecological findings should be confirmed at the individual level or in animal models.

\section{Conclusion}

The mean concentration of Mn in drinking water in Huai'an city was much higher than the concentration according to the national standards for drinking water quality. And the ratio of superscale is very high. Mn concentration is carcinogenic to people, which correlates with cancer incidence and mortality. Measures for mental pollution prevention and control must be taken immediately.

\section{Conflict of Interests}

The authors declare that there is no conflict of interests regarding the publication of this paper.

\section{Authors' Contribution}

Qin Zhang analysed the data and drafted the paper. Enchun Pan, Qiujin Xu, Linfei Liu, Wei Hu, and Yuan He conducted the investigation and participated in discussing. Qiujin Xu and Cunzhen Liang were responsible for laboratory testing. Enchun Pan supervised the project and revised the paper. All authors read and approved the final paper.

\section{Acknowledgments}

This work was supported by State Environmental Protection Commonweal Trade Scientific Research (no. 200909054) and Jiangsu Province Preventive Medicine Foundation (no. YZ201019). We also thanked SU Ming, YUAN Shouguo, HE Shiling from Huai'an, Xuyi, and Jinhu CDC for epidemiological survey and sample collection.

\section{References}

[1] J. Ferlay, H. Shin, F. Bray, D. Forman, C. Mathers, and D. M. Parkin, "Estimates of worldwide burden of cancer in 2008: GLOBOCAN 2008," International Journal of Cancer, vol. 127, no. 12, pp. 2893-2917, 2010.

[2] C. Wanqing, Z. Siwei, Z. Rongshou et al., "Report of cancer incidence and mortality in China, 2009," China Cancer, vol. 22, no. 1, pp. 2-12, 2013 (Chinese).

[3] C. Heidelberger, "Chemical carcinogenesis," Cancer, vol. 40, supplement S1, pp. 430-433, 1977.

[4] E. Pan, W. Hu, M. Wu, and J. Zhou, "Analysis and comparison of the malignant tumors death cause of residents between the two counties in Huai'an city during 30 years," Zhonghua Zhong Liu Za Zhi, vol. 16, no. 16, pp. 1210-1212, 2009 (Chinese).

[5] G. Yuan, Q. Li, Y. Du et al., "Analyses on cancer incidence and mortality in Huaian area, China, from 2009 to 2011," The Chinese-German Journal of Clinical Oncology, vol. 11, no. 9, pp. 497-503, 2012.

[6] K. M. Erikson, T. Syversen, J. L. Aschner, and M. Aschner, "Interactions between excessive manganese exposures and dietary iron-deficiency in neurodegeneration," Environmental Toxicology and Pharmacology, vol. 19, no. 3, pp. 415-421, 2005.

[7] Agency for Toxic Substances and Disease Registry (ATSDR), Toxicological profile for Manganese (Draft for Public Comment), U.S. Department of Health and Human Services, Public Health Service, Atlanta, Ga, USA, 2008.

[8] G. M. Solomon, A. M. Huddle, E. K. Silbergeld, and J. Herman, "Manganese in gasoline: are we repeating history? New solutions," Winter, pp. 17-25, 1997. 
[9] Federal Register US Federal Register, "Contaminants on the drinking water contaminant candidate list," Federal Register, vol. 68, no. 138, pp. 42897-42906, 2003.

[10] IARC, Surgical Implants and Other Foreign Bodies. IARC Monographs on the Evaluation of Carcinogenic Risks to Humans, vol. 74, IARC, Lyon, France, 1999.

[11] A. B. Bowman, G. F. Kwakye, E. H. Hernández, and M. Aschner, "Role of manganese in neurodegenerative diseases," Journal of Trace Elements in Medicine and Biology, vol. 25, no. 4, pp. 191203, 2011.

[12] J. G. Spangler and J. C. Reid, "Environmental manganese and cancer mortality rates by county in north carolina: an ecological study," Biological Trace Element Research, vol. 133, no. 2, pp. 128$135,2010$.

[13] T. Chuang, J. Liu, C. Lin et al., "Human manganese superoxide dismutase suppresses HER2/neu-mediated breast cancer malignancy," FEBS Letters, vol. 581, no. 23, pp. 4443-4449, 2007.

[14] J. Zejnilovic, N. Akev, H. Yilmaz, and T. Isbir, "Association between manganese superoxide dismutase polymorphism and risk of lung cancer," Cancer Genetics and Cytogenetics, vol. 189, no. 1, pp. 1-4, 2009.

[15] N. Joo, S. Kim, Y. Jung, and K. Kim, "Hair iron and other minerals' level in breast cancer patients," Biological Trace Element Research, vol. 129, no. 1-3, pp. 28-35, 2009.

[16] E. Kilic, R. Saraymen, A. Demiroglu, and E. Ok, "Chromium and manganese levels in the scalp hair of normals and patients with breast cancer," Biological Trace Element Research, vol. 102, no. 1-3, pp. 19-25, 2004.

[17] World Health Organization, Manganese in drinking-water: Background document for development of WHO Guidelines for Drinking-water Quality, 2004, http://www.who.int/water_ sanitation_health/dwq/chemicals/manganese.pdf.

[18] R. P. Hanzlik, P. Bhatia, R. Stitt, and G. J. Traiger, "Biotransformation and excretion of methylcyclopentadienyl manganese tricarbonyl in the rat," Drug Metabolism and Disposition, vol. 8, no. 6, pp. 428-433, 1980.

[19] G. D. Stoner, M. B. Shimkin, M. C. Troxell, T. L. Thompson, and L. S. Terry, "Test for carcinogenicity of metallic compounds by the pulmonary tumor response in strain A mice," Cancer Research, vol. 36, no. 5, pp. 1744-1747, 1976.

[20] D. Hafeman, P. Factor-Litvak, Z. Cheng, A. van Geen, and H. Ahsan, "Association between manganese exposure through drinking water and infant mortality in Bangladesh," Environmental Health Perspectives, vol. 115, no. 7, pp. 1107-1112, 2007.

[21] L. A. Gottschalk, T. Rebello, M. S. Buchsbaum, H. G. Tucker, and E. L. Hodges, "Abnormalities in hair trace elements as indicators of aberrant behavior," Comprehensive Psychiatry, vol. 32, no. 3, pp. 229-237, 1991. 


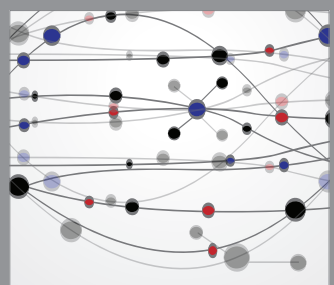

The Scientific World Journal
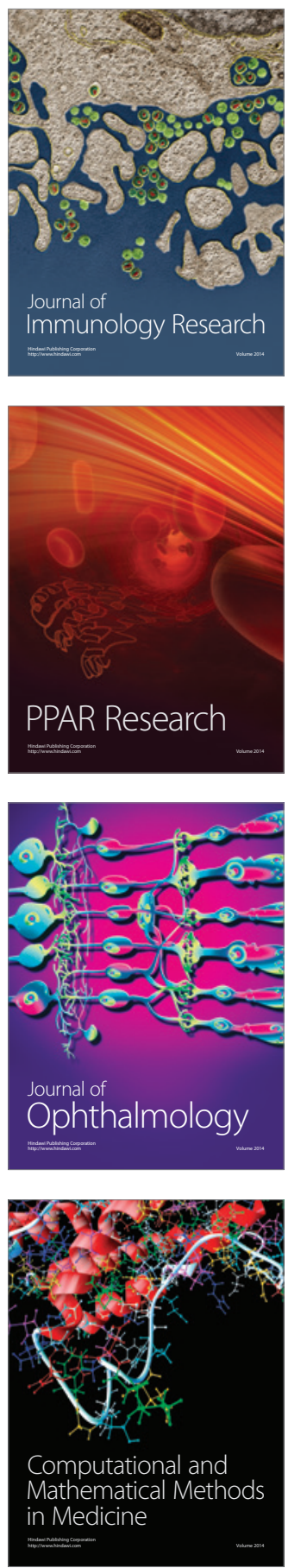

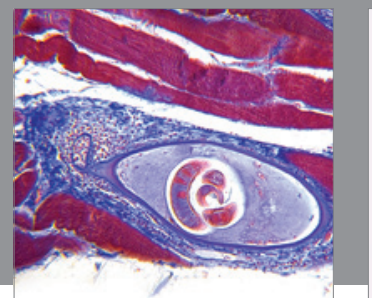

Gastroenterology

Research and Practice
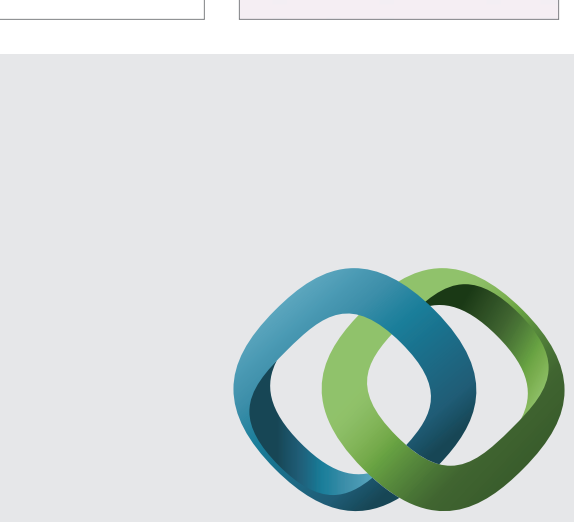

\section{Hindawi}

Submit your manuscripts at

http://www.hindawi.com
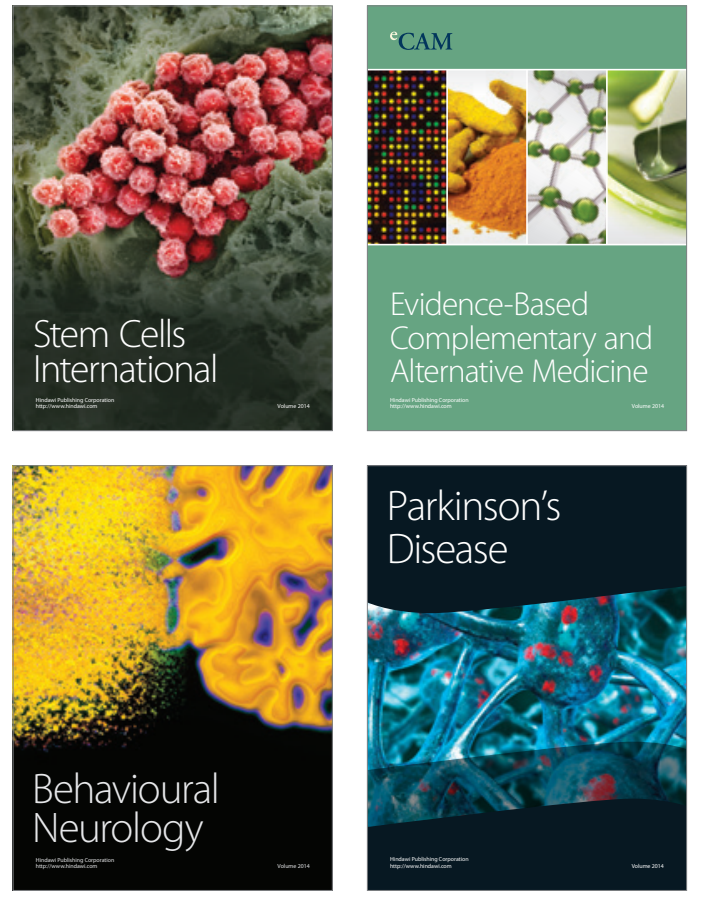
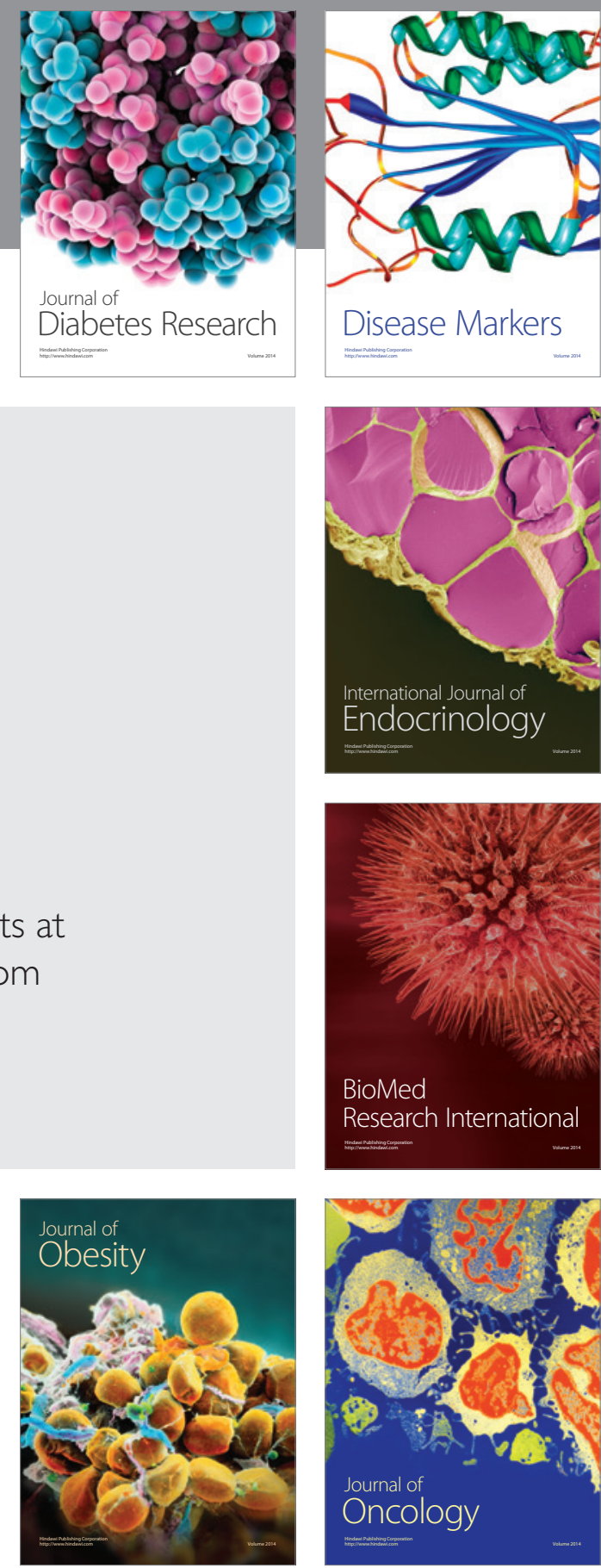

Disease Markers
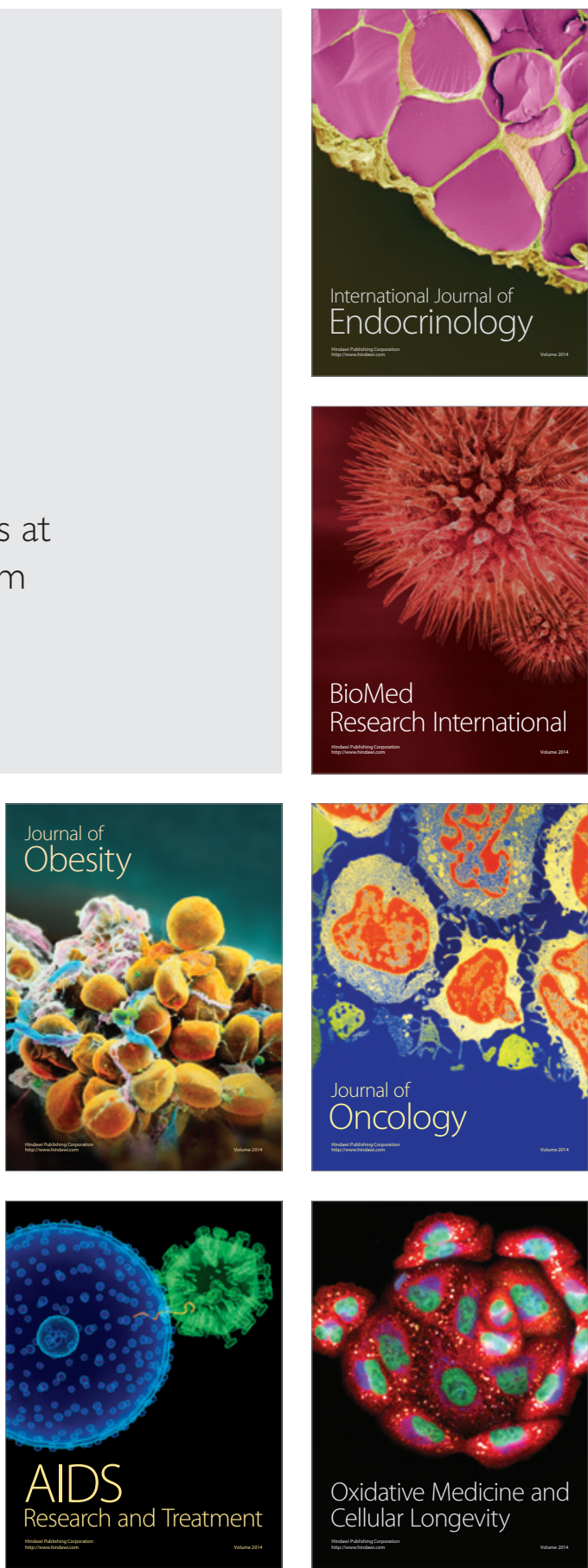